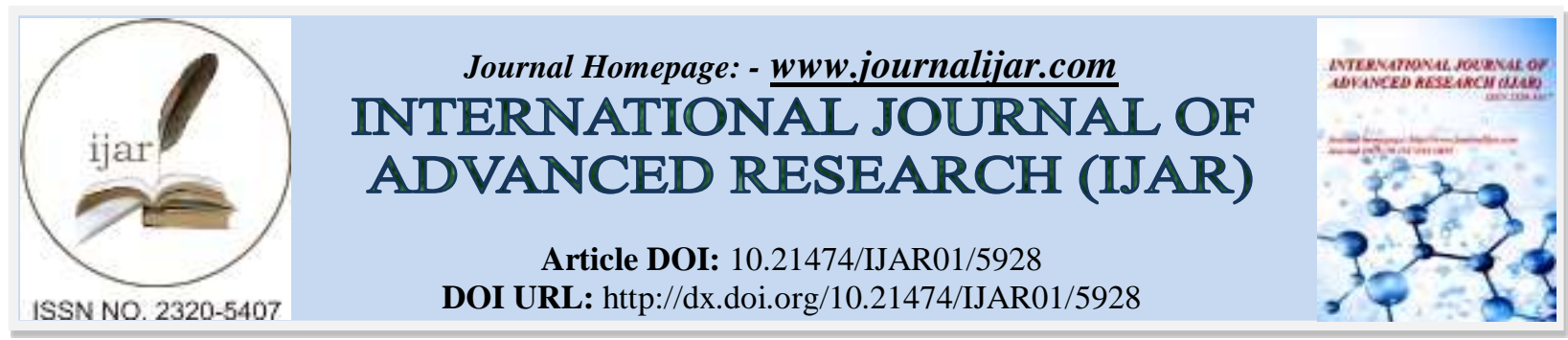

RESEARCH ARTICLE

\title{
A RETROSPECTIVE STUDY EVALUATED THE SAFETY AND CLINICAL AND ANATOMIC EFFECTIVENESS OF ENDOVENOUS LASER THERAPY (EVLT) AND MICROPHLEBECTOMY IN THE TREATMENT OF VARICOSE VEINS SECONDARY TO SAPHENOUS REFLUX IN IRAQ.
}

Saadoon H Al Hayazie.

FICMS, cardiothoracic and vascular surgeon.

\section{Manuscript Info}

Manuscript History

Received: 21 September 2017

Final Accepted: 23 October 2017

Published: November 2017

\section{Abstract}

Introduction: Chronic venous disorders (CVDs) of the lower extremity are common problems, which is commonly the result of reflux in one or more of the saphenous veins and their primary tributaries. Treatment options in patients with saphenous vein incompetence include conservative management or elimination of these incompetent pathways using endovenous techniques or surgery.

Method: From Jan 15, 2015, to Jan 15, 2017, 350 EVLT procedures were performed in 350 patients (213 women) with a mean age of 40.8 years (range, 18-75 years). A 1470-nm diode laser(venacure laser device ,Angiodynamics) and microphlebectomy were used. All sites of superficial axial reflux above and below the knee were ablated. Symptoms of venous insufficiency were present in $97 \%$, and 10 patients $(2.85 \%)$ had an open ulcer when they underwent operation. Patients had clinical follow-up visits, including duplex ultrasound examination, at 1 week, 1 month, 3 months, 6 months and were assessed for deep venous thrombosis (DVT), recanalization of the ablated vein, nerve injury, ulcer healing, and resolution of symptoms.

Results: A total of 310 great saphenous veins (88.57\%), 30 small saphenous veins $(8.57 \%), 10$ combined great and short saphenous veins (2.8) were ablated by endovenous laser therapy by using venacure laser device (1470),Angiodynamics. An average of 280 combined micro phlebectomies $(80 \%)$ were performed per case treated. The primary ablation rate at 1 and 6 months was $98.2 \%$. Recanalization occurred in 3 veins $(0.85 \%)$; in this group 2 were successfully closed with a second EVLT.. Postoperatively, the 9 ulcers showed healing at a mean of 5.2 weeks (range, 2-10 weeks), and only 1 failure of healing. No major complicatioens occurred. One DVT $(0.28 \%)$ occurred, but no pulmonary emboli ,one( $0.28 \%$ ) skin burns had occured. Local transient paresthesia at the ankle and midcalf level occurred in 3 patients $(0.85 \%)$.

Conclusions: EVLT of all sites of superficial axial reflux above and below the knee and microphlebectomy demonstrated that the combined approach is safe and effective at eliminating reflux, affording symptomatic relief, and healing ulcers. It offers the additional advantage of resolving varicose veins and its cause in just one visit, leading to immediate better cosmetic results. 
Copy Right, IJAR, 2017,. All rights reserved.

\section{Introduction:-}

Venous insufficiency is a genetically influenced, chronic, and progressive disorder. The ultimate goal of any treatment regimen is to eliminate sources of reflux in order to control symptoms and progression of disease, improve cosmesis, promote ulcer healing, and prevent recurrence or a combination of these. The best therapeutic results are based on two hemodynamic principles: the abolishment of the highest point of reflux and the elimination of the incompetent and dilated venous segments.

Endovenous laser treatment (EVLT) allows delivery of laser energy directly into the vein lumen. Published reports confirm that endovenous laser ablation of an incompetent great saphenous vein (GSV)1, 2, 3, or small saphenous vein (SSV), 4 is safe 5 and can provide outcomes equal to or better than traditional surgical ligation and stripping.6, Some practitioners advocate ablating the thigh segment of the GSV and treating the remaining visible varicosities with sclerotherapy during subsequent follow-up visits. Our chosen protocol is to perform EVLT of all refluxing truncal veins, above and below the knee, and to remove all varicose veins with microphlebectomies (MPs) at the same time. This approach mirrors past protocols in which the incompetent GSV was stripped in combination with stab avulsion of varicosities. Several large series have included GSV, SSV, EVLT ablations, but studies specifically of the success rate and risks associated with EVLT combined with MP have included only a small number of patients.7, 8 .

The purpose of this study is to report the safety and clinical and anatomic effectiveness of the combined application of EVLT and MP in a large number of patients .

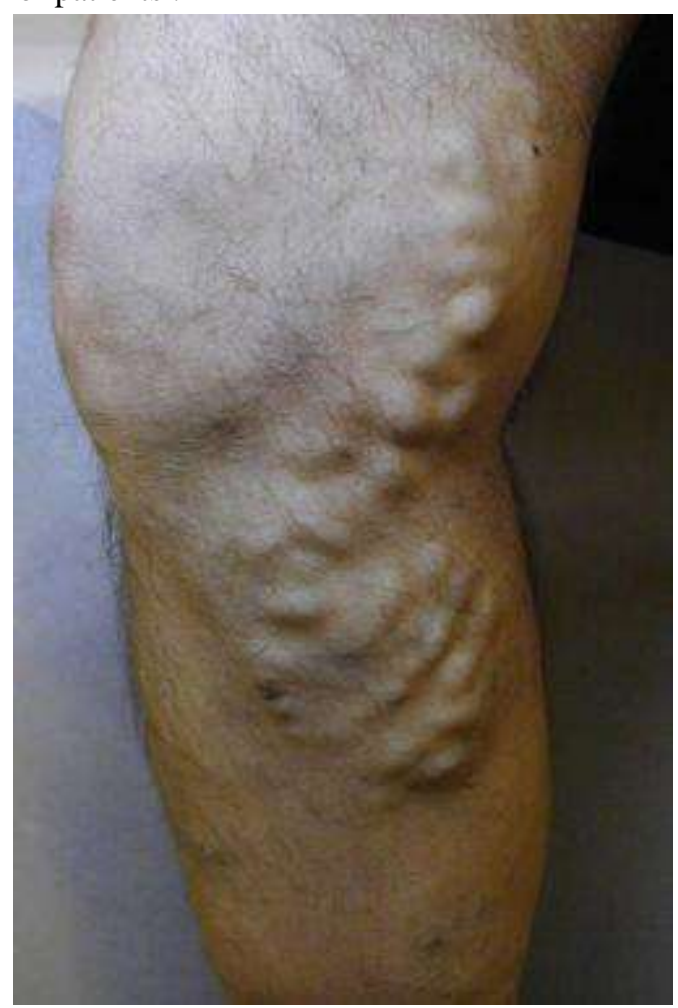

Fig 1:- patient with varicose vein preoperatively

Materials and methods:-

Patient selection and criteria:-

This retrospective study included 350 patients with venous insufficiency who presented for routine evaluation at the vein clinic and underwent EVLT ablations. Directed history and physical examination was performed to document varicosities and symptoms according to the CEAP classification. All patients had preoperative documentation of axial venous reflux using a Sonoscape duplex ultrasound (DUS) unit. 
Inclusion criteria included varicose veins caused by saphenous reflux (GSV and SSV) and their tributaries in patients aged $>14$ years. Exclusion criteria included nonpalpable pedal pulses, inability to ambulate, deep vein thrombosis (DVT), general poor health; pregnancy, and extremely tortuous veins that would not allow endovenous catheterization. After an initial examination and consultation, patients who met the selection criteria were offered the choice of surgery or EVLT. Nearly all patients chose EVLT rather than surgical ligation and stripping. .

\section{Technique:-}

All patients were treated as an ambulatory procedure dayclinic under local anesthesia. DUS imaging was done with the patient standing. All refluxing truncal veins were identified and marked in the skin, and a percutaneous entry point was chosen where reflux was no longer seen or where the vein became too small to access for ablation.

With the use of local anesthesia and sonographic guidance, the vein was punctured using the Seldinger technique. A 6F introducer sheath was placed into the vein over a guidewire $2 \mathrm{~cm}$ distal to saphenofemoral junction in case of GSV ablation (fig 2 .fig 3), and $2 \mathrm{~cm}$ distal to saphenopopliteal junction in the SSV cases, accessing at the ankle level if needed to ablate the entire length of a refluxing vein.

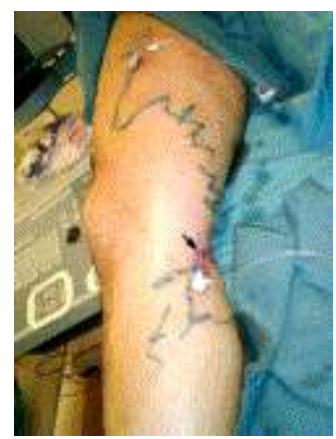

Fig 2:- inroducing sheathes in the GSV at lower thigh.

The intraluminal position within the vein was confirmed by aspiration of nonpulsatile venous blood and visualization with ultrasound. A $600-\mu \mathrm{m}$ laser fiber was introduced into the sheath and advanced to the first site . Position was confirmed by direct visualization of the red aiming beam of the laser fiber through the skin.

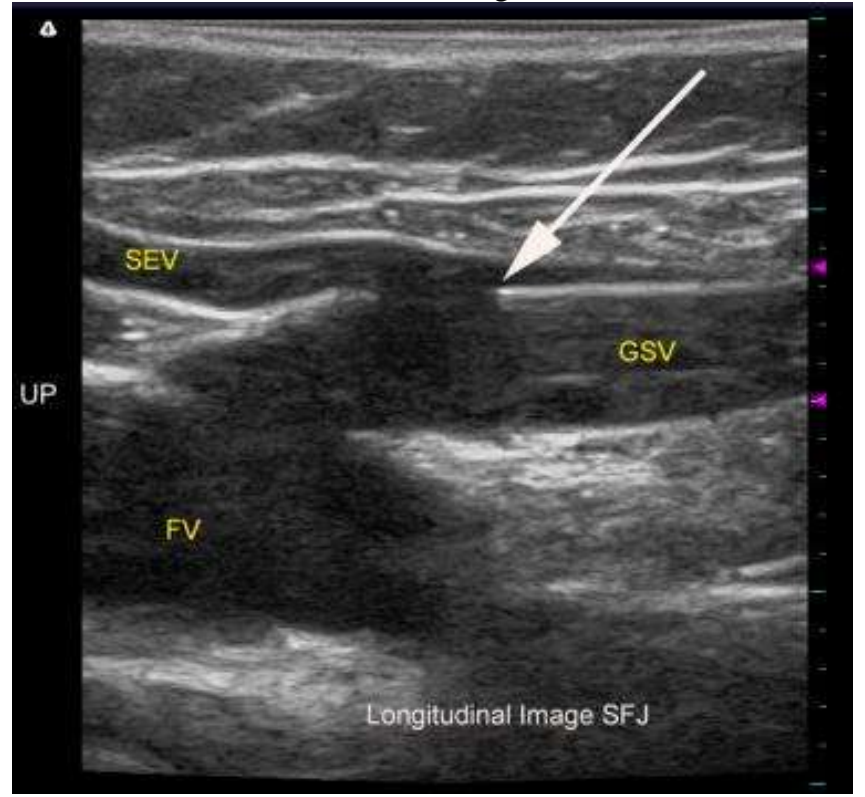

Fig 3:- (sagittal) duplex ultrasound image of the saphenofemoral junction during the positioning of the tip of a laser fiber during an endovenous laser ablation. The laser tip is in the greater saphenous vein (GSV) just beyond the superficial epigastric vein (SEV) origin and is marked by the arrow. FV=femoral vein. 
Tumescent local anesthesia, consisting of 200 to $400 \mathrm{~mL}$ of $0.1 \%$ lidocaine neutralized with sodium bicarbonate, was administered along the perivenous space under ultrasound guidance.

The tip of the laser fiber was repositioned within the GSV or SSV, $2 \mathrm{~cm}$ distal to the SFJ or SPJ, respectively. Laser energy was delivered using an 1470-nm diode laser (venacure 1470, angiodynamics) at 8-10 W in continuous mode. The vein was treated from $2 \mathrm{~cm}$ below the SFJ or SPJ to approximately $1 \mathrm{~cm}$ above the skin entry site. The laser fiber was withdrawn at an average rate of $2 \mathrm{~mm} / \mathrm{s}$ for GSV and SSV. These rates of withdrawal represent about 60 $\mathrm{J} / \mathrm{cm}$ and $70 \mathrm{~J} / \mathrm{cm}$ of energy delivered, respectively. These steps were repeated as needed to treat all refluxing truncal veins.

All patients underwent concomitant MP an 11 scalpel was used to make a 2-mm skin incision, and a hook device was used to remove the refluxing tributaries immediately after the laser ablation.

Once the procedure was completed, compression pads were applied over the treated area, and a low-stretch elastic compression bandage was placed on the treated limb. In the open ulcer cases confeel dressing was used and changed weekly until complete healing was achieved.

All patients were encouraged to ambulate the day of the procedure, to resume their normal daily activities in 2 to 3 days, and to wear the elastic bandage 24 hours a day for 7 days and then to replace it with a 15 to $20 \mathrm{~mm} \mathrm{Hg}$, fullthigh or pantyhose graduated support stocking for at least 1 month at all times, except to sleep or shower. Nonsteroidal anti-inflammatory drugs and an oral second-generation cephalosporin were ordered for the first week after therapy.

\section{Follow-up protocol:-}

Patients had clinical follow-up visits, including DUS examination at 1 week, 1 month, 3 months, 6 months . At the time of the visit, patients were asked about the severity of immediate postprocedural pain; timing to resume daily normal activities, work and exercise; complaints of nerve injury, and about changes in their extremity symptoms compared with those before the procedure. The presence of residual varicosities, edema, skin changes, and ulceration was recorded. The DUS scans assessed for the presence of DVT and for saphenous ablation or recanalization, defined as the absence or presence of flow, respectively, in a previously ablated vein. At the 3-month visit, patients were asked to complete a patient satisfaction questionnaire, a nonvalidated instrument that we created, asking about relief of symptoms of chronic venous insufficiency, cosmetic results, and overall satisfaction.

\section{Results:-}

Endovenous laser ablations in 350 patients who also underwent MP for treatment of varicose veins during a 24month period at day clinic . Symptoms were present in $97 \%$ of patients, who had a median CEAP clinical class of C 3.6 (range, 2-6). Open ulcers were present in 10 patients (2.85\%) at operation. Demographics and CEAP clinical classification of the studied population are summarized in Table I .

Table I:- Patient characteristics and disease severity scores by $\mathrm{C}$ category of the CEAP classification.

\begin{tabular}{|l|l|l|}
\hline Characteristics & No. $(\%)$ & Means (SD) \\
\hline Patients & 350 & \\
\hline Age, years & $(14-75)$ & $40.8(12.6)$ \\
\hline Weight, kg & $(45-150)$ & $72.4(15.2)$ \\
\hline Height, cm & $(145-180)$ & $164(8.7)$ \\
\hline Body mass index & $(20-32)$ & $26.5(4.6)$ \\
\hline Sex & & \\
\hline Female & $213(60.85)$ & \\
\hline Male & $137(39.142)$ & \\
\hline CEAP & & \\
\hline C2 & $116.2(33.2)$ & \\
\hline C3 & $59.15(16.9)$ & \\
\hline C4 & $123.55(35.3)$ & \\
\hline C5 & $28.35(8.1)$ & \\
\hline C6 & $22.75(6.5)$ & \\
\hline
\end{tabular}


Figure I:-

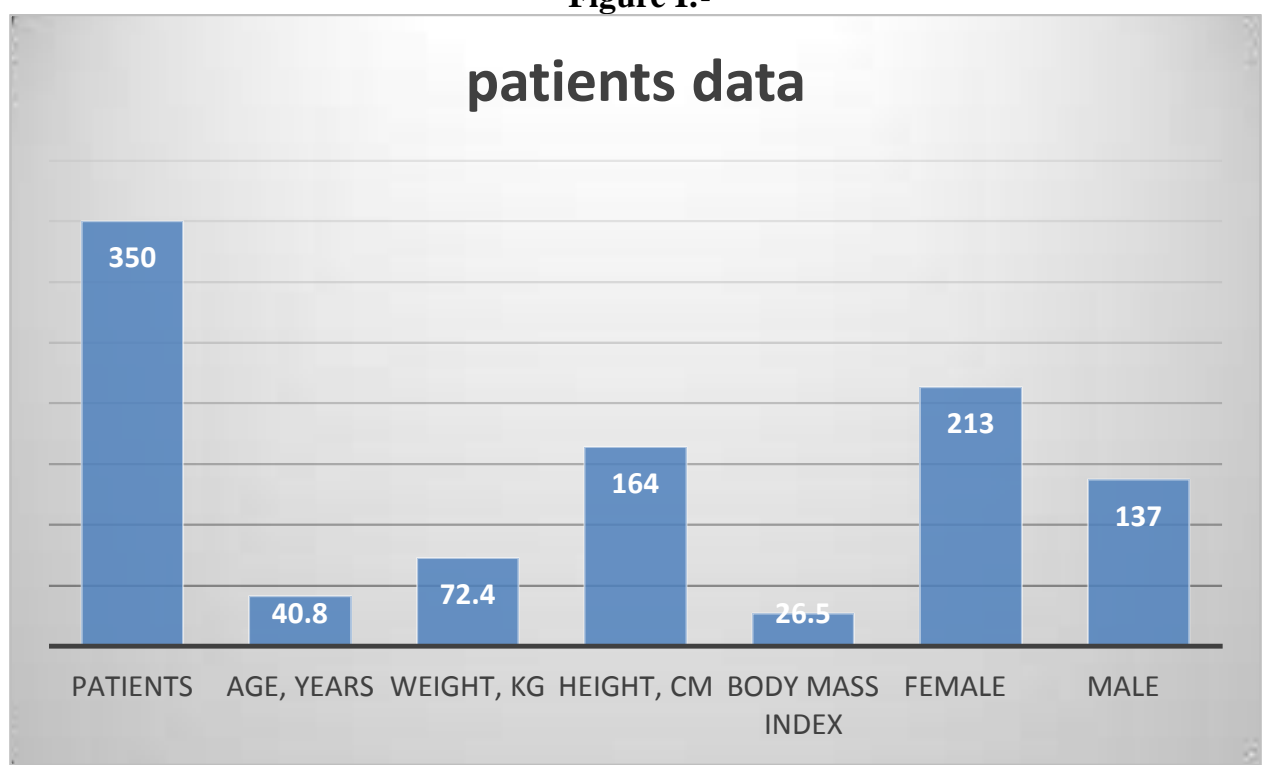

Figure II:- CEAP clinical classification of the patients included by the study

\section{CEAP clinical classification}

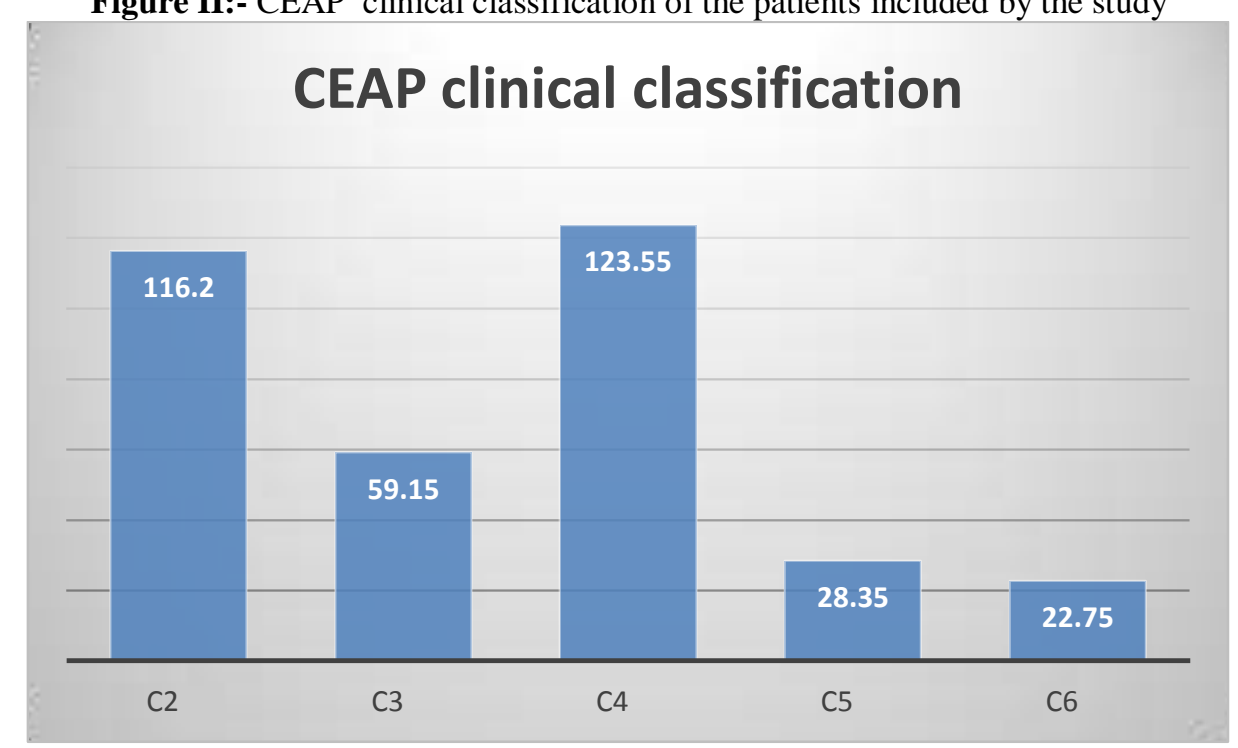

SD, Standard deviation:-

Ablation was performed in 310 GSVs (88.57\%), 30 SSVs (8.57\%), 10 patient combined GSV and SSV abaltion $(2.8 \%)$. Of the GSVs treated, $70.2 \%$ were ablated to the above the knee level, $20.4 \%$ to the knee, $8.82 \%$ below the knee, and $0.57 \%$ to the ankle level. Pretreatment vein diameter, measured in the upright position 1 to $2 \mathrm{~cm}$ below the SFJ and SPJ, was a mean of $7.5 \mathrm{~mm}$ (range, 4-15 mm), and a mean of $6.8 \mathrm{~mm}$ (range, 4-12 mm) for the GSV and SSV cases, respectively. Vein characteristics of the entire series are reported in Table IITable II. Patients underwent 280 MPs .

Table II:- Vein details and laser energy density

\begin{tabular}{|l|l|l|l|}
\hline Characteristics & GSV & SSV & \\
\hline Veins, N. & 315 & 45 & \\
\hline Diameter ,mm & 7.5 & 6.8 & \\
\hline Treated length, cm & $33.04(13.7)$ & $15.8(6.7)$ & \\
\hline Total energy, J & 1700 & 560 & \\
\hline Laser energy, J/cm & 70.4 & 60.2 & \\
\hline
\end{tabular}




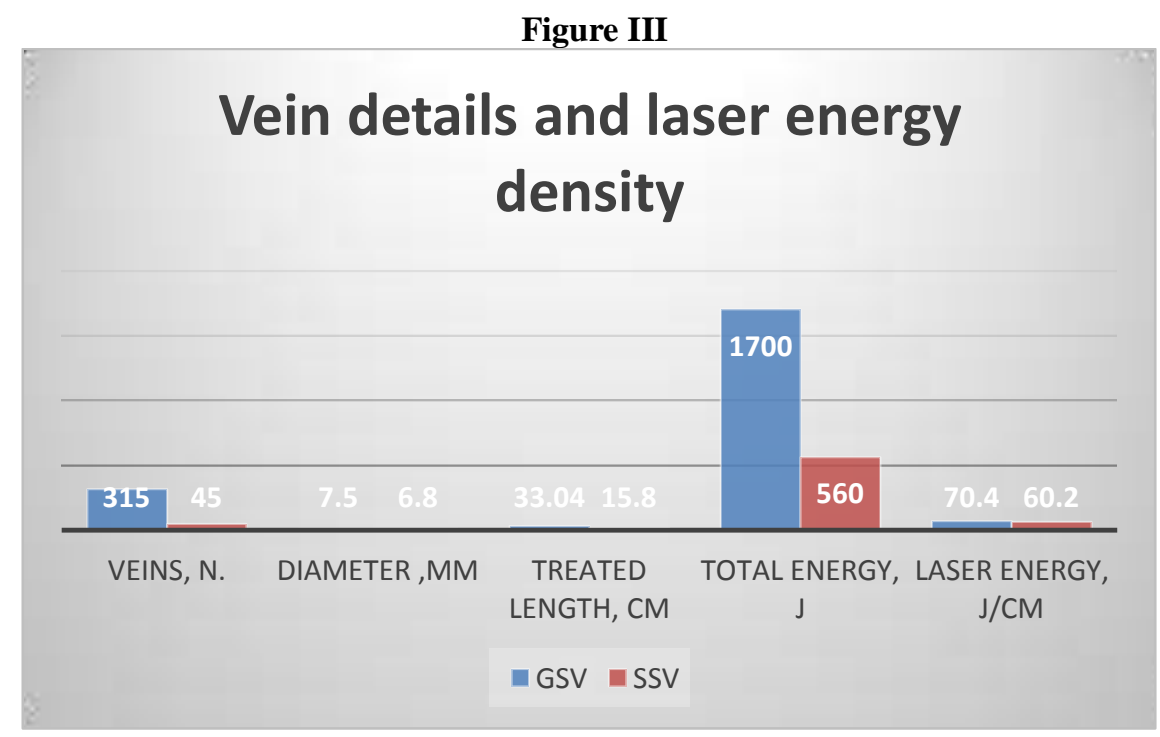

GSV, Great saphenous vein; SSV, small saphenous vein.

Continuous data are presented as means (standard deviation).

The primary ablation rate for the veins treated during the study period was $99.43 \%$ at 1 months and $98.2 \%$ at 6 months,

During the study period, 3 veins recanalized $(0.85 \%)$. Of these, symptoms of venous insufficiency (anatomic and clinical failure) developed, and they were successfully treated with a second EVLT.

The 10 limbs with an open ulcer that were operated on showed healing at a mean of 5.2 weeks (range, 2-10 weeks) after the procedure, and only one reopened during the study period .

Mild to moderate pain along the course of the treated vein during the first week and ecchymoses along the area of tumescent anesthesia administration and at phlebectomy sites were noted in most patients, as well as indurations. Superficial phlebitis of associated tributary varicose veins was noted in 10 patients (2.9\%) and resolved with compression therapy and over-the-counter nonsteroidal anti-inflammatory medication in all cases. Local transient paresthesia at the ankle and midcalf level occurred in 9 patients $(2.43 \%)$ and resolved spontaneously after 2 weeks. Most of these patients underwent extensive MP below the knee. Hyperpigmentation occurred in 14 patients (4\%) and cellulitis in $3(1 \%)$. Only one case $(0.28 \%)$ of DVT was found in the GSV group. the patient had ileofemoral involvement and was successfully treated after a 6-month course of oral anticoagulation, with the GSV remaining occlud. No patients presented with pulmonary embolism, one case of mild skin burn.

\section{Discussion:-}

Although GSV reflux is the most common underlying cause of varicose veins, other sources of superficial reflux often contribute to or are the sole cause of significant lower extremity varicosities. For instance, the SSV incompetence has been associated with the entire spectrum of signs and symptoms of chronic venous disease, including ulceration.Furthermore, our early experience demonstrated that EVLT of residual reflux below the knee was required to control symptoms in a few patients.

The clinical significance of persistent below the knee (BK) GSV (BK-GSV) reflux after EVLT has been studied by other investigators. Theivacumar et al19 evaluated 69 limbs after EVLT of the thigh GSV and found BK-GSV reflux of $>1$ second duration in 28 (41\%). Although the Aberdeen Varicose Vein Symptom Severity scores improved in this latter group of patients, the change was significantly less compared with the patients in whom no reflux was found in the untreated BK-GSV. Those 28 patients also needed more sclerotherapy to complete 
treatment.9 Similar results have been observed by Timperman,10 who also reported that symptomatic relief is obtained safely and effectively with additional EVLT of the BK-GSV.

Improved understanding of the mechanism of action of EVLT and its high degree of safety and effectiveness in the treatment of GSV reflux has led to the exploration of treatment of non-GSV sources of superficial reflux, with excellent results.5 Therefore, by targeting all the sources and length of axial reflux, above and below the knee, we can provide patients with a safe and superior alternative to ablating just the proximal portion of a refluxing saphenous vein, and this is the rationale behind our approach.

Combining MP with EVLT offers the additional advantage of resolving the varicose veins in just one visit, leading to immediate better cosmetic results. Both procedures can be done simultaneously, under local anesthesia and conscious sedation, without a significant increase of complications. Our rate of postoperative pain, cellulitis, or paresthesias is not very different from series where EVLT was the only procedure performed. None of our patients found the paresthesias or numbness to be a significant concern. We did have a smaller number of patients with postoperative phlebitis compared with those series.

Ulcer healing occurred at a mean of 5.2 weeks after treatment in the 10 patients who underwent the procedure with an open ulcer. The ulcer reopened in only one patients who underwent a second EVLT due to recanalization of the ablated vein .

The primary ablation rate for the 350 veins treated during the years 2015 to 2017 of $99.26 \%$ at 1 months and $98.25 \%$ at 6 months is very similar to published reports. Desmyttere et al11 reported a primary ablation rate of $97 \%$ at 4 years in 511 GSVs treated with a 980-nm endovenous laser. During the study period, 3 veins recanalized (1.76\%), representing an anatomic failure; however, these veins were noticeably smaller, and $57 \%$ of patients were symptomfree, without recurrent varicose veins. As a possible explanation, it is likely that shrinking a refluxing vein to a smaller diameter allowed the valves to completely or partially close, thus decreasing or eliminating reflux. Only 2 patients in this group presented with symptoms of venous insufficiency, indicating anatomic and clinical failure, and they were successfully treated with a second EVLT. Only one patient, with BMI of $31 \mathrm{~kg} / \mathrm{m} 2$, presented with an anatomic failure after his second EVLT, reflecting the importance of obesity as an independent risk factor for recanalization.

We observed that a BMI $>30 \mathrm{~kg} / \mathrm{m} 2$ and a vein diameter $\geq 8.5 \mathrm{~mm}$ were independent risk factors for recanalization. Timperman 12 has also found that obesity was common among patients in whom recanalization occurred. It is known that obese patients have increased abdominal and femoral venous pressure.13 In this latter group of failures, it is possible that the fibrosis caused by the EVLT was overcome by increased femoral venous pressure, with the saphenous vein reopening in a proximal to distal fashion, sometimes into a varicose tributary close to the SFJ that functions similarly to a relief valve. MP of these varicose tributary at the time of EVLT and ligation of the SFJ to isolate the GSV from increased venous pressure may improve the success of the endovenous ablation. These findings have relevance in counseling patients before the procedure about their risk of recanalization and also may suggest which patients should have a prophylactic ligation of the saphenous vein to reduce this undesired outcome.

\section{Conclusion:-}

The combined approach of EVLT of all sites of superficial axial reflux, above and below the knee, with microphlebectomy was demonstrated to be safe and effective at eliminating reflux, affording symptomatic relief, and healing ulcers. It offers the additional advantage of resolving varicose veins and their cause in just one visit, leading to immediate better cosmetic results. Further investigations may ascertain whether ligating the saphenous vein at the SFJ or SPJ in patients with vein diameters $>8.5 \mathrm{~mm}$ or with BMIs $>30 \mathrm{~kg} / \mathrm{m} 2$, will reduce the rate of recanalization, thus improving the long-term results of EVLT. 


\section{References:-}

1. Navarro, L., Min, R.J., and Bone, C. Endovenous laser: a new minimally invasive method of treatment for varicose veins-preliminary observations using an $810 \mathrm{~nm}$ diode laser. Dermatol Surg. 2001; 27: 117-122.

2. Min, R.J., Khilnani, N.M., and Zimmet, S.E. Endovenous laser treatment of saphenous vein reflux: long-term results. J Vasc Interv Radiol. 2003; 14: 991-996

3. Perkowski, P., Ravi, R., Gowda, R.C., Olsen, D., Ramaiah, V., Rodríguez-Lopez, J.A. et al. Endovenous laser ablation of the saphenous vein for treatment of venous insufficiency and varicose veins: early results from a large single-center experience. J Endovasc Ther. 2004; 11: 132-138

4. Proebstle, T.M., Gul, D., and Kargl, A. Endovenous laser treatment of the lesser saphenous vein with a 940-nm Diode Laser: Early results. Dermatol Surg. 2003; 29: 357-361

5. Gibson, K.D., Ferris, B.L., Polissar, N., Neradilek, B., and Pepper, D. Endovenous laser treatment of the short saphenous vein: Efficacy and complications. J Vasc Surg. 2007; 45: 795-803

6. Medeiros, C.A.F. and Lucas, G.C. Comparison of endovenous treatment with an $810 \mathrm{~nm}$ laser versus conventional stripping of the great saphenous vein in patients with primary varicose veins. Dermatol Surg. 2005; 31: 1685-1694

7. Mekako, A., Hatfield, J., Bryce, J., Heng, M., Lee, D., McCollum, P.T. et al. Combined endovenous laser therapy and ambulatory phlebectomy: refinement of a new technique. Eur J Endovasc Surg. 2006; 32: 725-729

8. Sadick, N.S. and Wasser, S. Combined endovascular laser with ambulatory phlebectomy for the treatment of superficial venous incompetence: a 4-year perspective. J Cosmet Laser Ther. 2007; 9: 9-13

1. 16-Ramelet, A.A. Phlebectomy: technique, indications and complications. Int Angiol. 2002; 21: 45-61

9. Theivacumar, N., Dellagrammaticas, D., Mavor, A., and Gough, M.J. Clinical significance of persistent belowknee great saphenous vein reflux following endovenous ablation: do we need to modify treatment? (European Venous Forum tripartite meeting abstracts). Phlebology. 2006; 21: 14

10. Timperman, P.E. Endovenous laser treatment of incompetent below-knee great saphenous veins. J Vasc Interv Radiol. 2007; 18: 1495-1499

11. Desmyttere, J., Grard, C., Wassmer, B., and Mordon, S. Endovenous 980-nm laser treatment of saphenous veins in a series of 500 patients. J Vasc Surg. 2007; 46: 1242-1247

12. Timperman, P.E. Prospective evaluation of higher energy great saphenous vein endovenous laser treatment. J Vasc Interv Radiol. 2005; 16: 791-794

13. Sugerman, H.J. Effects of increased intra-abdominal pressure in severe obesity. Surg Clin North Am. 2001; 81: 1063-1075. 